Helsinki University of Technology, Institute of Mathematics, Research Reports Teknillisen korkeakoulun matematiikan laitoksen tutkimusraporttisarja

\title{
A POSTERIORI ESTIMATES FOR THE STOKES EIGENVALUE PROBLEM
}

Carlo Lovadina Mikko Lyly Rolf Stenberg 

Helsinki University of Technology, Institute of Mathematics, Research Reports Teknillisen korkeakoulun matematiikan laitoksen tutkimusraporttisarja

\section{A POSTERIORI ESTIMATES FOR THE STOKES EIGENVALUE PROBLEM}

Carlo Lovadina Mikko Lyly Rolf Stenberg 
Carlo Lovadina, Mikko Lyly, and Rolf Stenberg: A posteriori estimates for the Stokes eigenvalue problem; Helsinki University of Technology, Institute of Mathematics, Research Reports A511 (2007).

Abstract: We consider the Stokes eigenvalue problem. For the eigenvalues we derive both upper and lower a-posteriori error bounds. The estimates are verified by numerical computations.

AMS subject classifications: $65 \mathrm{~N} 30,34 \mathrm{~L} 15$

Keywords: Stokes eigenvalue problem. A-posteriori error estimate. Adaptive computations.

\section{Correspondence}

Dipartimento di Matematica, Università di Pavia, and IMATI-CNR

Via Ferrata 1, Pavia I-27100, Italy

carlo.lovadina@unipv.it

CSC - Scientific Computing Ltd.

P.O. Box 405, FIN-02101 Espoo, Finland

mikko.lyly@csc.fi

Institute of Mathematics, Helsinki University of Technology

P.O.Box 1100, 02015 TKK, Finland

rolf.stenberg@hut.fi

ISBN-13 978-951-22-8456-6

ISBN-10 951-22-8456-1

Helsinki University of Technology

Department of Engineering Physics and Mathematics

Institute of Mathematics

P.O. Box 1100, FI-02015 TKK, Finland

email:math@tkk.fi http://math.tkk.fi/ 


\section{Introduction}

Regarding a posteriori analysis for finite element methods, most of the results in the literature are addressed to source problems (for example, see [1], [8] and [18], and the references therein). On the contrary, only few results are known about the a posteriori error analysis for eigenvalue problems. We mention here, in a non-exhaustive way, the work [14] for self-adjoint elliptic problems, and the generalisation detailed in [13] to elliptic operators, non necessarily self-adjoint. Moreover, a simple and elegant analysis for the Laplace operator has been performed in [10], while a mixed method has been considered in [9], by exploiting its equivalence with an approximation of nonconforming type (see [2]).

In this paper we present an a posteriori error analysis for the finite element discretization of the Stokes eigenvalue problem, introducing and studying a suitable residual-based error indicator. An outline of the paper is as follows. In Section 2 we briefly recall the eigenvalue problem for the Stokes operator, as well as its finite element discretization. In particular, we focus on stable schemes, which provide reliable approximation for both the source and the eigenvalue problem (see [4]). In Section 3 we introduce the residual-based error indicator. Following the guidelines of [10], we show that the error indicator is equivalent to error, up to higher order terms. Finally, in Section 4 we present some numerical tests for the MINI element, which is a stable element (see [5] and [6], for example), and thus it falls into the category of methods considered. As expected, the numerical experiments confirm our theoretical predictions.

Throughout the paper we will use standard notation for Sobolev norms and seminorms. Moreover, we will denote with $C$ a generic positive constant independent of the mesh parameter $h$.

\section{The Stokes eigenvalue problem and its finite element discretization}

Let $\Omega \subset \mathbb{R}^{N}(N=2,3)$ be a Lipschitz domain, with boundary $\Gamma$. We are interested in the eigenvalue problem for the Stokes system with homogeneous boundary conditions, i.e.:

$$
\begin{cases}\text { Find }(\boldsymbol{u}, p ; \lambda), \text { with } \boldsymbol{u} \neq \mathbf{0} \text { and } \lambda \in \mathbb{R}, \text { such that } & \\ -\Delta \boldsymbol{u}+\nabla p=\lambda \boldsymbol{u} & \text { in } \Omega \\ \operatorname{div} \boldsymbol{u}=0 & \text { in } \Omega, \\ \boldsymbol{u}=\mathbf{0} & \text { on } \Gamma .\end{cases}
$$

By introducing the bilinear form

$$
\mathcal{B}(\boldsymbol{u}, p ; \boldsymbol{v}, q):=(\nabla \boldsymbol{u}, \nabla \boldsymbol{v})-(\operatorname{div} \boldsymbol{v}, p)-(\operatorname{div} \boldsymbol{u}, q),
$$


and setting $\boldsymbol{V}=\left[H_{0}^{1}(\Omega)\right]^{N}$ and $P=L_{0}^{2}(\Omega)$, Problem (1) can be written in a variational form as follows:

$$
\left\{\begin{array}{l}
\text { Find }(\boldsymbol{u}, p ; \lambda) \in(\boldsymbol{V} \times P) \times \mathbb{R}, \text { with } \boldsymbol{u} \neq \mathbf{0}, \text { such that } \\
\mathcal{B}(\boldsymbol{u}, p ; \boldsymbol{v}, q)=\lambda(\boldsymbol{u}, \boldsymbol{v}) \quad \forall(\boldsymbol{v}, q) \in \boldsymbol{V} \times P .
\end{array}\right.
$$

We recall (see, e.g. [5]) that the bilinear form $\mathcal{B}$ is stable, i.e.:

- Given $(\boldsymbol{v}, q) \in \boldsymbol{V} \times P$, there exists $(\boldsymbol{w}, s) \in \boldsymbol{V} \times P$ such that

$$
\left\{\begin{array}{l}
\|\boldsymbol{w}\|_{1}+\|s\|_{0} \leq C \\
\|\boldsymbol{v}\|_{1}+\|q\|_{0} \leq \mathcal{B}(\boldsymbol{v}, q ; \boldsymbol{w}, s)
\end{array}\right.
$$

and it is continuous, i.e.:

- For every $(\boldsymbol{v}, q),(\boldsymbol{w}, s) \in \boldsymbol{V} \times P$, it holds

$$
\mathcal{B}(\boldsymbol{v}, q ; \boldsymbol{w}, s) \leq C\left(\|\boldsymbol{v}\|_{1}+\|q\|_{0}\right)\left(\|\boldsymbol{w}\|_{1}+\|s\|_{0}\right) .
$$

We now turn to the discretization of Problem (3) by finite elements. Let $\left\{\mathcal{C}_{h}\right\}_{h>0}$ be a sequence of decompositions of $\Omega$ into elements $K$, satisfying the usual compatibility conditions (see [7]). We also assume that the family $\left\{\mathcal{C}_{h}\right\}_{h>0}$ is regular, i.e. there exists a constant $\sigma>0$ such that

$$
h_{K} \leq \sigma \rho_{K} \quad \forall K \in \mathcal{C}_{h},
$$

where $h_{K}$ is the diameter of the element $K$ and $\rho_{K}$ is the maximum diameter of the circles contained in $K$. Associated with the mesh $\mathcal{C}_{h}$, we select finite elements spaces $\boldsymbol{V}_{h} \subset \boldsymbol{V}$ and $P_{h} \subset P$, and we consider the discrete Stokes eigenvalue problem:

$$
\left\{\begin{array}{l}
\text { Find }\left(\boldsymbol{u}_{h}, p_{h} ; \lambda_{h}\right) \in\left(\boldsymbol{V}_{h} \times P_{h}\right) \times \mathbb{R}, \text { with } \boldsymbol{u}_{h} \neq \mathbf{0}, \text { such that } \\
\mathcal{B}\left(\boldsymbol{u}_{h}, p_{h} ; \boldsymbol{v}, q\right)=\lambda_{h}\left(\boldsymbol{u}_{h}, \boldsymbol{v}\right) \quad \forall(\boldsymbol{v}, q) \in \boldsymbol{V}_{h} \times P_{h} .
\end{array}\right.
$$

We assume that the pair $\left(\boldsymbol{V}_{h}, P_{h}\right)$ satisfies the following properties:

- (Inf-sup condition) There exists $\beta>0$ independent of $h$, such that

$$
\sup _{\boldsymbol{v}_{h} \in \boldsymbol{V}_{h}} \frac{\left(\operatorname{div} \boldsymbol{v}_{h}, q_{h}\right)}{\left\|\boldsymbol{v}_{h}\right\|_{1}} \geq \beta\left\|q_{h}\right\|_{0} \quad \forall q_{h} \in P_{h} .
$$

- Assuming that $\boldsymbol{u} \in\left[H^{1+r}(\Omega)\right]^{N}$ and $p \in H^{r}(\Omega)$, for some $r \in(0,1]$, it holds

$$
\inf _{\boldsymbol{v}_{h} \in \boldsymbol{V}_{h}}\left\|\boldsymbol{u}-\boldsymbol{v}_{h}\right\|_{1} \leq C h^{r}|\boldsymbol{u}|_{r+1}
$$

and

$$
\inf _{q_{h} \in P_{h}}\left\|p-q_{h}\right\|_{0} \leq C h^{s}|p|_{r}
$$


It is well-known (see [6], for instance) that (8)-(10) imply convergence and stability of the given finite element scheme for the Stokes source problem. It has been proved in [4] that (8)-(10) are sufficient conditions for the convergence of the Stokes eigenvalue problem (7) as well. Indeed, by using the regularity results detailed in, e.g., [12] and [16], and well-established techniques for eigenvalue approximation (see [3], [15] and [4], for example), one has the following result.

Theorem 2.1. Given an eigenpair $(\boldsymbol{u}, p ; \lambda) \in(\boldsymbol{V} \times P) \times \mathbb{R}$, solution of (3), there exists $r \in(0,1]$ such that $\boldsymbol{u} \in\left[H^{1+r}(\Omega)\right]^{N}, p \in H^{r}(\Omega)$. Furthermore, for every positive $h \leq h_{0}(\lambda)$, there exists a discrete eigenpair $\left(\boldsymbol{u}_{h}, p_{h} ; \lambda_{h}\right) \in$ $\left(\boldsymbol{V}_{h} \times P_{h}\right) \times \mathbb{R}$, solution of (7), such that

$$
\begin{gathered}
\left|\lambda-\lambda_{h}\right| \leq C\left(\left\|\boldsymbol{u}-\boldsymbol{u}_{h}\right\|_{1}+\left\|p-p_{h}\right\|_{0}\right)^{2}, \\
\left\|\boldsymbol{u}-\boldsymbol{u}_{h}\right\|_{1}+\left\|p-p_{h}\right\|_{0} \leq C h^{r}\left(\|\boldsymbol{u}\|_{1+r}+\|p\|_{r}\right), \\
\left\|\boldsymbol{u}-\boldsymbol{u}_{h}\right\|_{0} \leq C h^{2 r}\left(\|\boldsymbol{u}\|_{1+r}+\|p\|_{r}\right) .
\end{gathered}
$$

Throughout the rest of the paper, we will denote with

$$
e(\boldsymbol{u})=\boldsymbol{u}-\boldsymbol{u}_{h}, \quad e(p)=p-p_{h}
$$

the eigenfunction errors, where $\boldsymbol{u}, \boldsymbol{u}_{h}, p$ and $p_{h}$ are as in Theorem 2.1.

\section{A posteriori error analysis}

The aim of this section is to introduce a suitable residual-based error estimator for the Stokes eigenvalue problems. To begin, for each element $K \in \mathcal{C}_{h}$ we introduce the residuals (cf. (1))

$$
\begin{gathered}
R_{K, 1}\left(\boldsymbol{u}_{h}, p_{h}\right)=\Delta \boldsymbol{u}_{h}-\nabla p_{h}+\lambda_{h} \boldsymbol{u}_{h}, \\
R_{K, 2}\left(\boldsymbol{u}_{h}\right)=\operatorname{div} \boldsymbol{u}_{h}, \\
R_{\partial K}\left(\boldsymbol{u}_{h}, p_{h}\right)=\llbracket\left(\nabla \boldsymbol{u}_{h}-p_{h} \boldsymbol{I}\right) \cdot \boldsymbol{n}_{K} \rrbracket_{\mid \partial K} .
\end{gathered}
$$

Accordingly, we define the local error estimator as

$$
\eta_{K}^{2}=h_{K}^{2}\left\|R_{K, 1}\left(\boldsymbol{u}_{h}, p_{h}\right)\right\|_{0, K}^{2}+\left\|R_{K, 2}\left(\boldsymbol{u}_{h}\right)\right\|_{0, K}^{2}+\frac{h_{K}}{2}\left\|R_{\partial K}\left(\boldsymbol{u}_{h}, p_{h}\right)\right\|_{0, \partial K}^{2} .
$$

Finally, the global error estimator is given by

$$
\eta^{2}=\sum_{K \in \mathcal{C}_{h}} \eta_{K}^{2}
$$




\subsection{Upper bounds}

We now provide an upper bound for our error estimator.

Theorem 3.1. Let $(\boldsymbol{u}, p ; \lambda) \in(\boldsymbol{V} \times P) \times \mathbb{R}$ be a solution of (3), and let $\left(\boldsymbol{u}_{h}, p_{h} ; \lambda_{h}\right) \in\left(\boldsymbol{V}_{h} \times P_{h}\right) \times \mathbb{R}$ be a solution of $(7)$, as in Theorem 2.1. For every positive $h \leq h_{0}(\lambda)$, it holds

$$
\|e(\boldsymbol{u})\|_{1}+\|e(p)\|_{0} \leq C\left(\eta+\left|\lambda-\lambda_{h}\right|+\lambda\left\|\boldsymbol{u}-\boldsymbol{u}_{h}\right\|_{0}\right) .
$$

Proof. Choose a generic pair $(\boldsymbol{v}, q) \in \boldsymbol{V}_{h} \times P_{h}$ as a test function for (3). By subtracting (7) from (3), we get the following error equation

$$
\mathcal{B}(e(\boldsymbol{u}), e(p) ; \boldsymbol{v}, q)=\left(\lambda \boldsymbol{u}-\lambda_{h} \boldsymbol{u}_{h}, \boldsymbol{v}\right) \quad \forall(\boldsymbol{v}, q) \in \boldsymbol{V}_{h} \times P_{h},
$$

where $e(\boldsymbol{u})$ and $e(p)$ are defined as in (14). By the stability of the continuous Stokes problem (cf. (4)), there exists $(\boldsymbol{w}, s) \in \boldsymbol{V} \times P$, with

$$
\|\boldsymbol{w}\|_{1}+\|s\|_{0} \leq C
$$

such that

$$
\|e(\boldsymbol{u})\|_{1}+\|e(p)\|_{0} \leq \mathcal{B}(e(\boldsymbol{u}), e(p) ; \boldsymbol{w}, s) .
$$

Let $\boldsymbol{w}^{I} \in \boldsymbol{V}_{h}$ be the Clément interpolant of $\boldsymbol{w}$ (cf. e.g. [5, 17]), and let $s^{I} \in$ $P_{h}$ be the $L^{2}$-projection of $s$. By using the error equation (7), estimate (9) gives

$$
\begin{aligned}
\|e(\boldsymbol{u})\|_{1}+\|e(p)\|_{0} & \leq \mathcal{B}\left(e(\boldsymbol{u}), e(p) ; \boldsymbol{w}-\boldsymbol{w}^{I}, s-s^{I}\right)+\left(\lambda \boldsymbol{u}-\lambda_{h} \boldsymbol{u}_{h}, \boldsymbol{w}^{I}\right) \\
& =\mathcal{B}\left(e(\boldsymbol{u}), e(p) ; \boldsymbol{w}-\boldsymbol{w}^{I}, s-s^{I}\right)-\left(\lambda \boldsymbol{u}-\lambda_{h} \boldsymbol{u}_{h}, \boldsymbol{w}-\boldsymbol{w}^{I}\right) \\
& +\left(\lambda \boldsymbol{u}-\lambda_{h} \boldsymbol{u}_{h}, \boldsymbol{w}\right)
\end{aligned}
$$

Integrating by parts, using the continuous Stokes equations (1), and recalling (1)-(3) we obtain

$$
\begin{gathered}
\mathcal{B}\left(e(\boldsymbol{u}), e(p) ; \boldsymbol{w}-\boldsymbol{w}^{I}, s-s^{I}\right)-\left(\lambda \boldsymbol{u}-\lambda_{h} \boldsymbol{u}_{h}, \boldsymbol{w}-\boldsymbol{w}^{I}\right) \\
=\sum_{K \in \mathcal{C}_{h}}\left\{\left(R_{K, 1}\left(\boldsymbol{u}_{h}, p_{h}\right), \boldsymbol{w}-\boldsymbol{w}^{I}\right)_{K}+\left(R_{K, 2}\left(\boldsymbol{u}_{h}\right), s-s^{I}\right)_{K}\right. \\
\left.+\frac{1}{2}\left\langle R_{\partial K}\left(\boldsymbol{u}_{h}, p_{h}\right), \boldsymbol{w}-\boldsymbol{w}^{I}\right\rangle_{\partial K}\right\},
\end{gathered}
$$

where the brackets $\langle\cdot, \cdot\rangle_{\partial K}$ denote the $L^{2}$ inner product on the boundary $\partial K$. Applying the Cauchy-Schwarz inequality to Eq. (11), we obtain

$$
\begin{aligned}
& \mathcal{B}\left(e(\boldsymbol{u}), e(p) ; \boldsymbol{w}-\boldsymbol{w}^{I}, s-s^{I}\right)-\left(\lambda \boldsymbol{u}-\lambda_{h} \boldsymbol{u}_{h}\right) \\
& \leq C\left\{\sum_{K \in \mathcal{C}_{h}}\left(h_{K}^{-2}\left\|\boldsymbol{w}-\boldsymbol{w}^{I}\right\|_{0, K}^{2}+h_{K}^{-1}\left\|\boldsymbol{w}-\boldsymbol{w}^{I}\right\|_{0, \partial K}^{2}+\left\|s-s^{I}\right\|_{0, K}\right)\right\}^{1 / 2} \times \\
& \left\{\sum_{K \in \mathcal{C}_{h}}\left(h_{K}^{2}\left\|R_{K, 1}\left(\boldsymbol{u}_{h}, p_{h}\right)\right\|_{0, K}^{2}+\left\|R_{K, 2}\left(\boldsymbol{u}_{h}\right)\right\|_{0, \partial K}^{2}+\frac{h_{K}}{2}\left\|R_{\partial K}\left(\boldsymbol{u}_{h}, p_{h}\right)\right\|_{0, \partial K}^{2}\right)\right\}^{1 / 2}
\end{aligned}
$$


Since for the Clément interpolation it holds

$$
\left\{\sum_{K \in \mathcal{C}_{h}}\left(h_{K}^{-2}\left\|\boldsymbol{w}-\boldsymbol{w}^{I}\right\|_{0, K}^{2}+h_{K}^{-1}\left\|\boldsymbol{w}-\boldsymbol{w}^{I}\right\|_{0, \partial K}^{2}\right)\right\}^{1 / 2} \leq C\|\boldsymbol{w}\|_{1},
$$

and for the $L^{2}$ projection we have

$$
\left\|s^{I}\right\|_{0} \leq\|s\|_{0},
$$

the estimates (12), (8) and (5) give

$$
\mathcal{B}\left(e(\boldsymbol{u}), e(p) ; \boldsymbol{w}-\boldsymbol{w}^{I}, s-s^{I}\right)-\left(\lambda \boldsymbol{u}-\lambda_{h} \boldsymbol{u}_{h}, \boldsymbol{w}-\boldsymbol{w}^{I}\right) \leq C \eta
$$

It remains to estimate the term $\left(\lambda \boldsymbol{u}-\lambda_{h} \boldsymbol{u}_{h}, \boldsymbol{w}\right)$, see (10). We may write

$$
\begin{aligned}
\left(\lambda \boldsymbol{u}-\lambda_{h} \boldsymbol{u}_{h}, \boldsymbol{w}\right) & =\left(\left(\lambda-\lambda_{h}\right) \boldsymbol{u}_{h}, \boldsymbol{w}\right)+\lambda\left(\left(\boldsymbol{u}-\boldsymbol{u}_{h}\right), \boldsymbol{w}\right) \\
& \leq\left|\lambda-\lambda_{h}\right|\left\|\boldsymbol{u}_{h}\right\|_{0}\|\boldsymbol{w}\|_{0}+\lambda\left\|\boldsymbol{u}-\boldsymbol{u}_{h}\right\|_{0}\|\boldsymbol{w}\|_{0} \\
& \leq C\left(\left|\lambda-\lambda_{h}\right|+\lambda\left\|\boldsymbol{u}-\boldsymbol{u}_{h}\right\|_{0}\right)
\end{aligned}
$$

where we have used (8). Collecting (15) and (16), from (10) we get

$$
\|e(\boldsymbol{u})\|_{1}+\|e(p)\|_{0} \leq C\left(\eta+\left|\lambda-\lambda_{h}\right|+\lambda\left\|\boldsymbol{u}-\boldsymbol{u}_{h}\right\|_{0}\right),
$$

i.e. estimate (6).

Corollary 3.1. For the eigenvalue approximation, it holds

$$
\left|\lambda-\lambda_{h}\right| \leq C\left(\eta^{2}+\left|\lambda-\lambda_{h}\right|^{2}+\lambda^{2}\left\|\boldsymbol{u}-\boldsymbol{u}_{h}\right\|_{0}^{2}\right) .
$$

Proof. The assertion immediately follows by squaring estimate (6), and using the a priori bound (11) of Theorem 2.1.

Remark 3.1. In view of Theorem 2.1 the quantities $\left|\lambda-\lambda_{h}\right|+\lambda\left\|\boldsymbol{u}-\boldsymbol{u}_{h}\right\|_{0}$ in (6) and $\left|\lambda-\lambda_{h}\right|^{2}+\lambda^{2}\left\|\boldsymbol{u}-\boldsymbol{u}_{h}\right\|_{0}^{2}$ in (18) are both higher-order terms.

\subsection{Lower bounds}

Next, we show a local lower bound on the estimator. We denote with $\omega(K)$ the union of all elements having at least one edge (for $N=2$ ) - or one face (for $N=3$ ) - in common with $K$. Similarly, for a given edge (for $N=2$ ) $E$ - or face (for $N=3$ ) - the set $\omega(E)$ is the union of the elements which contain $E$.

Theorem 3.2. Let $(\boldsymbol{u}, p ; \lambda) \in(\boldsymbol{V} \times P) \times \mathbb{R}$ be a solution of (3), and let $\left(\boldsymbol{u}_{h}, p_{h} ; \lambda_{h}\right) \in\left(\boldsymbol{V}_{h} \times P_{h}\right) \times \mathbb{R}$ be a solution of (7), as in Theorem 2.1. For every positive $h \leq h_{0}(\lambda)$, it holds

$$
\begin{aligned}
\eta_{K} \leq C\left(\|\nabla e(\boldsymbol{u})\|_{0, \omega(K)}\right. & +\|e(p)\|_{0, \omega(K)} \\
& \left.+\sum_{K^{\prime} \subset \omega(K)} h_{K^{\prime}}^{1 / 2}\left(\left|\lambda-\lambda_{h}\right|+\lambda\left\|\boldsymbol{u}-\boldsymbol{u}_{h}\right\|_{0, K^{\prime}}\right)\right) .
\end{aligned}
$$


Proof. We set

$$
\boldsymbol{v}_{K}:=h_{K}^{2} b_{K} R_{K, 1}\left(\boldsymbol{u}_{h}, p_{h}\right),
$$

where $b_{K}$ denotes the standard bubble function of the element $K$. By recalling (1) and by usual scaling arguments, we get

$$
\begin{aligned}
C h_{K}^{2} & \left\|R_{K, 1}\left(\boldsymbol{u}_{h}, p_{h}\right)\right\|_{0, K}^{2} \leq\left(\Delta \boldsymbol{u}_{h}-\nabla p_{h}+\lambda_{h} \boldsymbol{u}_{h}, \boldsymbol{v}_{K}\right)_{K} \\
& =\left(\Delta\left(\boldsymbol{u}_{h}-\boldsymbol{u}\right)-\nabla\left(p_{h}-p\right)+\lambda_{h} \boldsymbol{u}_{h}-\lambda \boldsymbol{u}, \boldsymbol{v}_{K}\right)_{K} \\
& =-\left(\Delta e(\boldsymbol{u})-\nabla e(p)+\lambda_{h} \boldsymbol{u}_{h}-\lambda \boldsymbol{u}, \boldsymbol{v}_{K}\right)_{K},
\end{aligned}
$$

where we have used $-\Delta \boldsymbol{u}+\nabla p-\lambda \boldsymbol{u}=\mathbf{0}$. We have

$$
\begin{aligned}
-\left(\Delta e(\boldsymbol{u})-\nabla e(p), \boldsymbol{v}_{K}\right)_{K}=\left(\nabla e(\boldsymbol{u}), \nabla \boldsymbol{v}_{K}\right)_{K}-\left(e(p), \operatorname{div} \boldsymbol{v}_{K}\right)_{K} \\
\leq C\left(\|\nabla e(\boldsymbol{u})\|_{0, K}+\|e(p)\|_{0, K}\right)\left\|\nabla \boldsymbol{v}_{K}\right\|_{0, K}, \\
\leq C\left(\|\nabla e(\boldsymbol{u})\|_{0, K}+\|e(p)\|_{0, K}\right) h_{K}\left\|R_{K, 1}\left(\boldsymbol{u}_{h}, p_{h}\right)\right\|_{0, K} .
\end{aligned}
$$

Furthermore, it holds

$$
\begin{aligned}
\left(\lambda_{h} \boldsymbol{u}_{h}-\lambda \boldsymbol{u}, \boldsymbol{v}_{K}\right)_{K} & =\left(\left(\lambda_{h}-\lambda\right) \boldsymbol{u}_{h}, \boldsymbol{v}_{K}\right)+\lambda\left(\left(\boldsymbol{u}_{h}-\boldsymbol{u}\right), \boldsymbol{v}_{K}\right) \\
& \leq\left(\left|\lambda-\lambda_{h}\right|\left\|\boldsymbol{u}_{h}\right\|_{0, K}+\lambda\left\|\boldsymbol{u}-\boldsymbol{u}_{h}\right\|_{0, K}\right)\left\|\boldsymbol{v}_{K}\right\|_{0, K} \\
& \leq C\left(\left|\lambda-\lambda_{h}\right|+\lambda\left\|\boldsymbol{u}-\boldsymbol{u}_{h}\right\|_{0, K}\right)\left\|\boldsymbol{v}_{K}\right\|_{0, K} \\
& \leq C\left(\left|\lambda-\lambda_{h}\right|+\lambda\left\|\boldsymbol{u}-\boldsymbol{u}_{h}\right\|_{0, K}\right) h_{K}^{2}\left\|R_{K, 1}\left(\boldsymbol{u}_{h}, p_{h}\right)\right\|_{0, K} .
\end{aligned}
$$

From (21)-(23) we get

$$
\begin{aligned}
h_{K}\left\|R_{K, 1}\left(\boldsymbol{u}_{h}, p_{h}\right)\right\|_{0, K} & \leq C\left(\|\nabla e(\boldsymbol{u})\|_{0, K}+\|e(p)\|_{0, K}\right. \\
& \left.+h_{K}\left|\lambda-\lambda_{h}\right|+h_{K} \lambda\left\|\boldsymbol{u}-\boldsymbol{u}_{h}\right\|_{0, K}\right) .
\end{aligned}
$$

To continue, we trivially have

$$
\left\|R_{K, 2}\left(\boldsymbol{u}_{h}\right)\right\|_{0, K}=\left\|\operatorname{div} \boldsymbol{u}_{h}\right\|_{0, K}=\|\operatorname{div} e(\boldsymbol{u})\|_{0, K} \leq \sqrt{N}\|\nabla e(\boldsymbol{u})\|_{0, K} .
$$

Fix now an edge (for $N=2$ ) or a face (for $N=3$ ) $E \subset \partial K$. Consider

$$
\boldsymbol{\varphi}_{E}:=h_{E} b_{E} R_{E}\left(\boldsymbol{u}_{h}, p_{h}\right),
$$

where $h_{E}$ is the diameter of $E$, the function $b_{E} \in H_{0}^{1}(\omega(E))$ is the usual bubble function for $E$ (see [18], for example), and the residual $R_{E}\left(\boldsymbol{u}_{h}, p_{h}\right)$ is defined by (cf. also (3))

$$
R_{E}\left(\boldsymbol{u}_{h}, p_{h}\right)=\llbracket\left(\nabla \boldsymbol{u}_{h}-p_{h} \boldsymbol{I}\right) \cdot \boldsymbol{n}_{E} \rrbracket_{\mid E} \cdot
$$

By standard scaling arguments, using $\llbracket(\nabla \boldsymbol{u}-p \boldsymbol{I}) \cdot \boldsymbol{n}_{E} \rrbracket_{\mid E}=\mathbf{0}$, and integrating by parts, we get

$$
\begin{aligned}
C h_{E}\left\|R_{E}\left(\boldsymbol{u}_{h}, p_{h}\right)\right\|_{0, E}^{2} \leq & \left\langle\llbracket\left(\nabla \boldsymbol{u}_{h}-p_{h} \boldsymbol{I}\right) \cdot \boldsymbol{n}_{E} \rrbracket, \boldsymbol{\varphi}_{E}\right\rangle_{E} \\
= & -\left\langle\llbracket(\nabla e(\boldsymbol{u})-e(p) \boldsymbol{I}) \cdot \boldsymbol{n}_{E} \rrbracket, \boldsymbol{\varphi}_{E}\right\rangle_{E} \\
= & -\left(\nabla e(\boldsymbol{u}), \nabla \boldsymbol{\varphi}_{E}\right)_{\omega(E)}+\left(e(p), \operatorname{div} \boldsymbol{\varphi}_{E}\right)_{\omega(E)} \\
& \quad-\sum_{K \subset \omega(E)}\left(\Delta e(\boldsymbol{u})-\nabla e(p), \boldsymbol{\varphi}_{E}\right)_{K} .
\end{aligned}
$$


We also have, using again scaling arguments and (26):

$$
\begin{aligned}
-(\nabla e(\boldsymbol{u}), & \left.\nabla \boldsymbol{\varphi}_{E}\right)_{\omega(E)}+\left(e(p), \operatorname{div} \boldsymbol{\varphi}_{E}\right)_{\omega(E)} \\
& \leq C\left(\|\nabla e(\boldsymbol{u})\|_{0, \omega(E)}+\|e(p)\|_{0, \omega(E)}\right)\left\|\nabla \boldsymbol{\varphi}_{E}\right\|_{0, \omega(E)} \\
& \leq C\left(\|\nabla e(\boldsymbol{u})\|_{0, \omega(E)}+\|e(p)\|_{0, \omega(E)}\right) h_{E}^{1 / 2}\left\|R_{E}\left(\boldsymbol{u}_{h}, p_{h}\right)\right\|_{0, E} .
\end{aligned}
$$

Furthermore, it holds

$$
\begin{aligned}
-\sum_{K \subset \omega(E)} & \left(\Delta e(\boldsymbol{u})-\nabla e(p), \boldsymbol{\varphi}_{E}\right)_{K} \\
& =\sum_{K \subset \omega(E)}\left\{\left(R_{K, 1}\left(\boldsymbol{u}_{h}, p_{h}\right), \boldsymbol{\varphi}_{E}\right)_{K}+\left(\lambda \boldsymbol{u}-\lambda_{h} \boldsymbol{u}_{h}, \boldsymbol{\varphi}_{E}\right)_{K}\right\} .
\end{aligned}
$$

On the one hand we get

$$
\begin{aligned}
& \sum_{K \subset \omega(E)}\left(R_{K, 1}\left(\boldsymbol{u}_{h}, p_{h}\right), \boldsymbol{\varphi}_{E}\right)_{K} \\
& \quad \leq C\left(\sum_{K \subset \omega(E)} h_{K}\left\|R_{K, 1}\left(\boldsymbol{u}_{h}, p_{h}\right)\right\|_{0, K}\right) h_{E}^{1 / 2}\left\|R_{E}\left(\boldsymbol{u}_{h}, p_{h}\right)\right\|_{0, E} .
\end{aligned}
$$

Similar computations as in (23) show that

$$
\sum_{K \subset \omega(E)}\left(\lambda \boldsymbol{u}-\lambda_{h} \boldsymbol{u}_{h}, \boldsymbol{\varphi}_{E}\right) \leq C\left(\left|\lambda-\lambda_{h}\right|+\lambda\left\|\boldsymbol{u}-\boldsymbol{u}_{h}\right\|_{0, \omega(E)}\right) h_{E}^{3 / 2}\left\|R_{E}\left(\boldsymbol{u}_{h}, p_{h}\right)\right\|_{0, E} .
$$

Therefore, from (30), (31) and (32), we obtain

$$
\begin{aligned}
& -\sum_{K \subset \omega(E)}\left(\Delta e(\boldsymbol{u})-\nabla e(p), \boldsymbol{\varphi}_{E}\right)_{K} \\
& \leq C\left(\sum_{K \subset \omega(E)} h_{K}\left\|R_{K, 1}\left(\boldsymbol{u}_{h}, p_{h}\right)\right\|_{0, K}+h_{E}^{1 / 2}\left(\left|\lambda-\lambda_{h}\right|+\lambda\left\|\boldsymbol{u}-\boldsymbol{u}_{h}\right\|_{0, \omega(E)}\right)\right) \times \\
& \quad h_{E}^{1 / 2}\left\|R_{E}\left(\boldsymbol{u}_{h}, p_{h}\right)\right\|_{0, E} .
\end{aligned}
$$

Taking into account estimates (29) and (33), from (28) we infer

$$
\begin{aligned}
& h_{E}^{1 / 2}\left\|R_{E}\left(\boldsymbol{u}_{h}, p_{h}\right)\right\|_{0, E} \\
& \leq C\left(\sum_{K \subset \omega(E)} h_{K}\left\|R_{K, 1}\left(\boldsymbol{u}_{h}, p_{h}\right)\right\|_{0, K}+h_{E}^{1 / 2}\left(\left|\lambda-\lambda_{h}\right|+\lambda\left\|\boldsymbol{u}-\boldsymbol{u}_{h}\right\|_{0, \omega(E)}\right)\right) .
\end{aligned}
$$

Summing over the element edges (for $N=2$ ), or faces (for $N=3$ ), (34) and the regularity of the mesh $\mathcal{C}_{h}$ give

$$
\begin{aligned}
& \frac{h_{K}^{1 / 2}}{\sqrt{2}}\left\|R_{\partial K}\left(\boldsymbol{u}_{h}, p_{h}\right)\right\|_{0, \partial K} \\
& \leq C \sum_{K^{\prime} \subset \omega(K)}\left(h_{K^{\prime}}\left\|R_{K^{\prime}, 1}\left(\boldsymbol{u}_{h}, p_{h}\right)\right\|_{0, K^{\prime}}+h_{K^{\prime}}^{1 / 2}\left(\left|\lambda-\lambda_{h}\right|+\lambda\left\|\boldsymbol{u}-\boldsymbol{u}_{h}\right\|_{0, K^{\prime}}\right)\right) .
\end{aligned}
$$


By recalling (4), from (24), (25) and (35), we get

$$
\begin{aligned}
\eta_{K} \leq C\left(\|\nabla e(\boldsymbol{u})\|_{0, \omega(K)}\right. & +\|e(p)\|_{0, \omega(K)} \\
& \left.+\sum_{K^{\prime} \subset \omega(K)} h_{K^{\prime}}^{1 / 2}\left(\left|\lambda-\lambda_{h}\right|+\lambda\left\|\boldsymbol{u}-\boldsymbol{u}_{h}\right\|_{0, K^{\prime}}\right)\right),
\end{aligned}
$$

which completes the proof.

\section{Numerical results}

Our numerical examples will be given for the twodimensional problem with the linear triangular MINI-element (see [6], for instance) for which the velocity and pressure spaces are defined as

$$
\boldsymbol{V}_{h}=\left\{\boldsymbol{v} \in \boldsymbol{V} \mid \boldsymbol{v}_{\mid K} \in\left[P_{1}(K)\right]^{2} \oplus\left[P_{3}(K) \cap H_{0}^{1}(K)\right]^{2} \forall K \in \mathcal{C}_{h}\right\}
$$

and

$$
P_{h}=\left\{q \in P \cap H^{1}(\Omega) \mid q_{\mid K} \in P_{1}(K) \forall K \in \mathcal{C}_{h}\right\}
$$

where $P_{k}(K)$ is the space of polynomials of degree $k$ defined on $K \in \mathcal{C}_{h}$. All the computations have been performed with the open-source finite element software Elmer [11].

\subsection{Square domain}

In our first example we will consider the square $\Omega=(-1,1) \times(-1,1)$ with homogenous Dirichlet boundary conditions imposed on the velocity. The finite element mesh is obtained by dividing the domain into $2 N \times N$ triangles as shown in Figure 1.

In Table 1 we have tabulated the 10 smallest eigenvalues of the Stokes operator as a function of $N \in\{4,8, \ldots, 128\}$. Our reference solution is given in the last column of the table. The reference has been extrapolated from the numerical results by assuming that the error $\left|\lambda-\lambda_{h}\right|$ behaves as $C h^{r}$ for some constants $C$ and $r$ independent of $h=\sqrt{2 / N}$.

The relative error $\left|\lambda-\lambda_{h}\right| / \lambda$ with respect to the reference solution is shown in Table 2. In Table 3 we have tabulated the values our a posteriori error estimator $\eta$. Note that in both cases, the convergence rate is approximately $r \approx 2$, as suggested by Theorem 2.1 and Corollary 3.1 .

\subsection{L-shaped domain}

In our second example we remove the bottom left quadrant of the square, and consider the L-shaped domain $(-1,1) \times(-1,1) \backslash[-1,0] \times[-1,0]$, again with homogenous Dirichlet conditions for the velocity, see Figure 1. The results from the calculations are shown in Tables 4-6.

For the L-shaped domain, the convergence rates of the exact and estimated errors vary in the range $1.7 \leq r \leq 2$, depending on the regularity of 


\begin{tabular}{c|c|c|c|c|c|c|c} 
Mode $/ N$ & 4 & 8 & 16 & 32 & 64 & 128 & ref \\
\hline 1 & 18.403 & 14.377 & 13.400 & 13.164 & 13.105 & 13.091 & 13.086 \\
2 & 33.716 & 25.879 & 23.730 & 23.204 & 23.074 & 23.042 & 23.031 \\
3 & 41.929 & 27.676 & 24.143 & 23.304 & 23.099 & 23.048 & 23.031 \\
4 & 53.024 & 39.937 & 34.078 & 32.555 & 32.177 & 32.084 & 32.053 \\
5 & 79.801 & 47.721 & 40.783 & 39.087 & 38.669 & 38.566 & 38.532 \\
6 & 91.089 & 52.290 & 44.193 & 42.351 & 41.905 & 41.794 & 41.759 \\
7 & 125.123 & 59.801 & 50.673 & 48.214 & 47.597 & 47.444 & 47.393 \\
8 & 128.224 & 67.366 & 52.419 & 48.626 & 47.698 & 47.469 & 47.393 \\
9 & 152.065 & 81.101 & 66.333 & 62.742 & 61.869 & 61.652 & 61.583 \\
10 & 155.823 & 83.846 & 67.104 & 62.928 & 61.915 & 61.664 & 61.583
\end{tabular}

Table 1: Numerical eigenvalues $\lambda_{h}$ for $h=\sqrt{2 / N}$ and the extrapolated reference solution $\lambda$ for the unit square.

\begin{tabular}{c|c|c|c|c|c|c|c} 
Mode $/ N$ & 4 & 8 & 16 & 32 & 64 & 128 & rate \\
\hline 1 & 0.4063 & 0.0986 & 0.0240 & 0.0059 & 0.0015 & 0.0004 & 2.020 \\
2 & 0.4639 & 0.1236 & 0.0303 & 0.0075 & 0.0018 & 0.0005 & 2.018 \\
3 & 0.8205 & 0.2017 & 0.0482 & 0.0118 & 0.0029 & 0.0007 & 2.026 \\
4 & 0.6543 & 0.2460 & 0.0632 & 0.0157 & 0.0039 & 0.0010 & 2.012 \\
5 & 1.0710 & 0.2385 & 0.0584 & 0.0144 & 0.0036 & 0.0009 & 2.019 \\
6 & 1.1813 & 0.2522 & 0.0583 & 0.0142 & 0.0035 & 0.0008 & 2.036 \\
7 & 1.6401 & 0.2618 & 0.0692 & 0.0173 & 0.0043 & 0.0011 & 2.000 \\
8 & 1.7055 & 0.4214 & 0.1060 & 0.0260 & 0.0064 & 0.0016 & 2.026 \\
9 & 1.4693 & 0.3169 & 0.0771 & 0.0188 & 0.0046 & 0.0011 & 2.032 \\
10 & 1.5303 & 0.3615 & 0.0896 & 0.0218 & 0.0054 & 0.0013 & 2.034
\end{tabular}

Table 2: Errors $\left|\lambda-\lambda_{h}\right| / \lambda$ and the convergence rate $r$ for the unit square.

\begin{tabular}{c|c|c|c|c|c|c|c} 
Mode $/ N$ & 4 & 8 & 16 & 32 & 64 & 128 & rate \\
\hline 1 & 1.7204 & 0.1325 & 0.0996 & 0.0255 & 0.0065 & 0.0016 & 1.976 \\
2 & 3.2066 & 0.1360 & 0.1542 & 0.0394 & 0.0100 & 0.0025 & 1.979 \\
3 & 4.7796 & 0.1581 & 0.1821 & 0.0462 & 0.0117 & 0.0030 & 1.982 \\
4 & 5.7537 & 0.1498 & 0.2569 & 0.0657 & 0.0167 & 0.0042 & 1.977 \\
5 & 9.9275 & 0.1535 & 0.2652 & 0.0668 & 0.0169 & 0.0043 & 1.986 \\
6 & 13.1493 & 0.1669 & 0.2806 & 0.0694 & 0.0175 & 0.0044 & 1.998 \\
7 & 18.3303 & 0.1443 & 0.3406 & 0.0868 & 0.0220 & 0.0056 & 1.979 \\
8 & 18.3590 & 0.1632 & 0.4001 & 0.1002 & 0.0253 & 0.0064 & 1.990 \\
9 & 22.9004 & 0.1686 & 0.3991 & 0.0975 & 0.0245 & 0.0062 & 2.005 \\
10 & 5.3907 & 0.1446 & 0.4223 & 0.1028 & 0.0258 & 0.0065 & 2.006
\end{tabular}

Table 3: Estimated errors $\eta$ and the convergence rate $r$ for the unit square. 


\begin{tabular}{c|c|c|c|c|c|c|c} 
Mode $/ N$ & 4 & 8 & 16 & 32 & 64 & 128 & ref \\
\hline 1 & 61.122 & 43.131 & 35.216 & 33.086 & 32.461 & 32.257 & 32.1734 \\
2 & 89.147 & 46.312 & 39.322 & 37.608 & 37.172 & 37.058 & 37.0199 \\
3 & 127.47 & 53.878 & 44.962 & 42.704 & 42.137 & 41.993 & 41.9443 \\
4 & 139.01 & 65.379 & 53.182 & 50.024 & 49.242 & 49.048 & 48.9844 \\
5 & 189.86 & 79.876 & 62.313 & 57.198 & 55.895 & 55.553 & 55.4365 \\
6 & 195.10 & 100.55 & 78.836 & 72.010 & 70.223 & 69.733 & 69.5600 \\
7 & 205.99 & 108.18 & 79.029 & 72.682 & 71.143 & 70.760 & 70.6382 \\
8 & 213.28 & 124.04 & 93.381 & 85.350 & 83.245 & 82.683 & 82.4832 \\
9 & 213.85 & 127.10 & 93.951 & 86.028 & 84.086 & 83.599 & 83.4450 \\
10 & 215.09 & 143.23 & 103.93 & 92.914 & 90.176 & 89.490 & 89.2902
\end{tabular}

Table 4: Numerical eigenvalues $\lambda_{h}$ for $h=\sqrt{2 / N}$ and the extrapolated reference solution $\lambda$ for the L-shaped domain.

\begin{tabular}{c|c|c|c|c|c|c|c} 
Mode $/ N$ & 4 & 8 & 16 & 32 & 64 & 128 & rate \\
\hline 1 & 0.8998 & 0.3406 & 0.0946 & 0.0284 & 0.0089 & 0.0026 & 1.7214 \\
2 & 1.4081 & 0.2510 & 0.0622 & 0.0159 & 0.0041 & 0.0010 & 1.9650 \\
3 & 2.0390 & 0.2845 & 0.0719 & 0.0181 & 0.0046 & 0.0012 & 1.9864 \\
4 & 1.8378 & 0.3347 & 0.0857 & 0.0212 & 0.0053 & 0.0013 & 2.0130 \\
5 & 2.4248 & 0.4409 & 0.1240 & 0.0318 & 0.0083 & 0.0021 & 1.9596 \\
6 & 1.8048 & 0.4455 & 0.1334 & 0.0352 & 0.0095 & 0.0025 & 1.9525 \\
7 & 1.9161 & 0.5315 & 0.1188 & 0.0289 & 0.0071 & 0.0017 & 2.0329 \\
8 & 1.5857 & 0.5038 & 0.1321 & 0.0348 & 0.0092 & 0.0024 & 1.9228 \\
9 & 1.5628 & 0.5232 & 0.1259 & 0.0310 & 0.0077 & 0.0018 & 2.0182 \\
10 & 1.4089 & 0.6041 & 0.1640 & 0.0406 & 0.0099 & 0.0022 & 2.0612
\end{tabular}

Table 5: Errors $\left|\lambda-\lambda_{h}\right| / \lambda$ and the convergence rate $r$ for the L-shaped domain.

the corresponding eigenfunction (see Theorem 2.1 and the analysis of MINI element [6] for more details). Nevertheless, the tables show that estimator $\eta$ is optimal in the sense that it always has approximately the same convergence rate as the true error with respect to the reference solution.

\subsection{Adaptive refinement for the L-shaped domain}

The software Elmer [11] uses a error balancing strategy. First, a a coarse starting mesh is prescribed. Then, after computing the approximate solution and the corresponding error estimators, a complete remeshing is done by using a Delaunay triangulation. The refining-coarsening strategy is based on the local error indicators and on the assumption that the local error is of the form

$$
\eta_{K}=C_{K} h_{K}^{p_{K}}
$$




\begin{tabular}{c|c|c|c|c|c|c|c} 
Mode $/ N$ & 4 & 8 & 16 & 32 & 64 & 128 & rate \\
\hline 1 & 2.3135 & 0.2992 & 0.0606 & 0.0200 & 0.0067 & 0.0023 & 1.7058 \\
2 & 3.4751 & 0.2133 & 0.0164 & 0.0095 & 0.0026 & 0.0007 & 1.9496 \\
3 & 5.9479 & 0.2334 & 0.0136 & 0.0077 & 0.0020 & 0.0006 & 1.9413 \\
4 & 7.0110 & 0.2472 & 0.0126 & 0.0055 & 0.0009 & 0.0003 & 1.9805 \\
5 & 13.152 & 0.3049 & 0.0175 & 0.0129 & 0.0041 & 0.0015 & 1.9007 \\
6 & 15.547 & 0.3969 & 0.0139 & 0.0226 & 0.0072 & 0.0024 & 1.8516 \\
7 & 24.925 & 0.7347 & 0.0248 & 0.0075 & 0.0014 & 0.0004 & 1.9822 \\
8 & 25.973 & 0.6432 & 0.0155 & 0.0172 & 0.0050 & 0.0018 & 1.8920 \\
9 & 28.716 & 0.7081 & 0.0167 & 0.0120 & 0.0031 & 0.0008 & 1.9752 \\
10 & 35.779 & 0.6366 & 0.0157 & 0.0127 & 0.0038 & 0.0012 & 1.9608
\end{tabular}

Table 6: Estimated errors $\eta$ and the convergence rate $r$ for the L-shaped domain.

for some constants $C_{K}$ and $p_{K}$. The new mesh is then built with the aim of having the error uniformly distributed over the elements.

The stopping criteria for the adaptive process is either a given tolerance for the maximum local estimator or the number of refinement steps. Between two subsequent adaptive steps we have used the value 2 for the change of the relative local mesh density ratio. For the element size, neither a maximum nor a minimum have been prescribed.

The sequence of meshes is shown in Figure 2. In Figure 3 the error estimator is plotted as a function of the number of degrees of freedom for the adaptive scheme and the uniform refinement.

\section{Acknowledgements}

This work has been supported by TEKES, the National Technology Agency of Finland, in the context of the project KOMASI (decision number 40288/05).

\section{References}

[1] M. Ainsworth And J. T. Oden, A posteriori error estimation in finite element analysis, Pure and Applied Mathematics (New York), Wiley-Interscience [John Wiley \& Sons], New York, 2000.

[2] D. N. Arnold And F. BrezzI, Mixed and nonconforming finite element methods: implementation, postprocessing and error estimates, RAIRO Modél. Math. Anal. Numér., 19 (1985), pp. 7-32.

[3] I. BABUŠKa And J. Osborn, Eigenvalue problems, in Handbook of Numerical Analysis, Vol. II, North-Holland, Amsterdam, 1991, pp. 641787. 
[4] D. Boffi, F. Brezzi, And L. Gastaldi, On the convergence of eigenvalues for mixed formulations, Ann. Scuola Norm. Sup. Pisa Cl. Sci. (4), 25 (1997), pp. 131-154 (1998).

[5] D. Braess, Finite Elements. Theory, Fast Solvers, and Applications in Solid Mechanics, Cambridge University Press, 1997.

[6] F. Brezzi And M. Fortin, Mixed and hybrid finite element methods, vol. 15 of Springer Series in Computational Mathematics, SpringerVerlag, New York, 1991.

[7] P. G. Ciarlet, The finite element method for elliptic problems, vol. 40 of Classics in Applied Mathematics, Society for Industrial and Applied Mathematics (SIAM), Philadelphia, PA, 2002. Reprint of the 1978 original [North-Holland, Amsterdam; MR0520174 (58 \#25001)].

[8] W. DÖRfler And M. Ainsworth, Reliable a posteriori error control for nonconformal finite element approximation of Stokes flow, Math. Comp., 74 (2005), pp. 1599-1619 (electronic).

[9] R. G. Durán, L. Gastaldi, and C. Padra, A posteriori error estimators for mixed approximations of eigenvalue problems, Math. Models Methods Appl. Sci., 9 (1999), pp. 1165-1178.

[10] R. G. Durán, C. Padra, And R. Rodríguez, A posteriori error estimates for the finite element approximation of eigenvalue problems, Math. Models Methods Appl. Sci., 13 (2003), pp. 1219-1229.

[11] Elmer Finite ElEMENT SOFTwARE HOMEPAGE. http://www.csc.fi/elmer.

[12] E. B. Fabes, C. E. Kenig, and G. C. Verchota, The Dirichlet problem for the Stokes system on Lipschitz domains, Duke Math. J., 57 (1988), pp. 769-793.

[13] V. Heuveline and R. Rannacher, A posteriori error control for finite approximations of elliptic eigenvalue problems, Adv. Comput. Math., 15 (2001), pp. 107-138 (2002).

[14] M. G. LARson, A posteriori and a priori error analysis for finite element approximations of self-adjoint elliptic eigenvalue problems, SIAM J. Numer. Anal., 38 (2000), pp. 608-625 (electronic).

[15] B. Mercier, J. Osborn, J. Rappaz, and P.-A. Raviart, Eigenvalue approximation by mixed and hybrid methods, Math. Comp., 36 (1981), pp. 427-453.

[16] G. SAVARÉ, Regularity results for elliptic equations in Lipschitz domains, J. Funct. Anal., 152 (1998), pp. 176-201. 
[17] L. R. Scott And S. Brenner, The Mathematical Theory of Finite Element Methods, Springer-Verlag, 1994.

[18] R. Verfürth, A Review of A Posteriori Error Estimation and Adaptive Mesh-Refinement Techniques, Wiley-Teubner, 1996. 

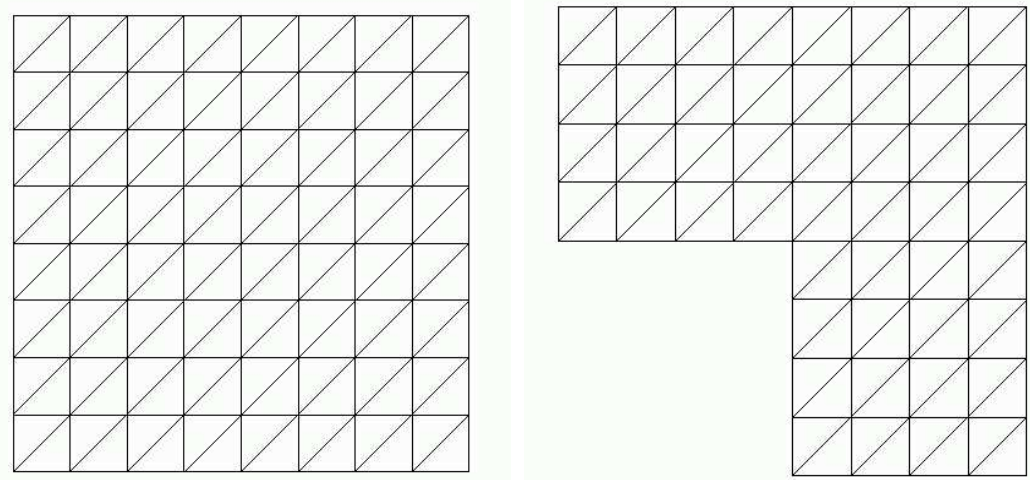

Figure 1: Uniform finite element partitioning of the unit square and L-shaped domain for $N=8$.
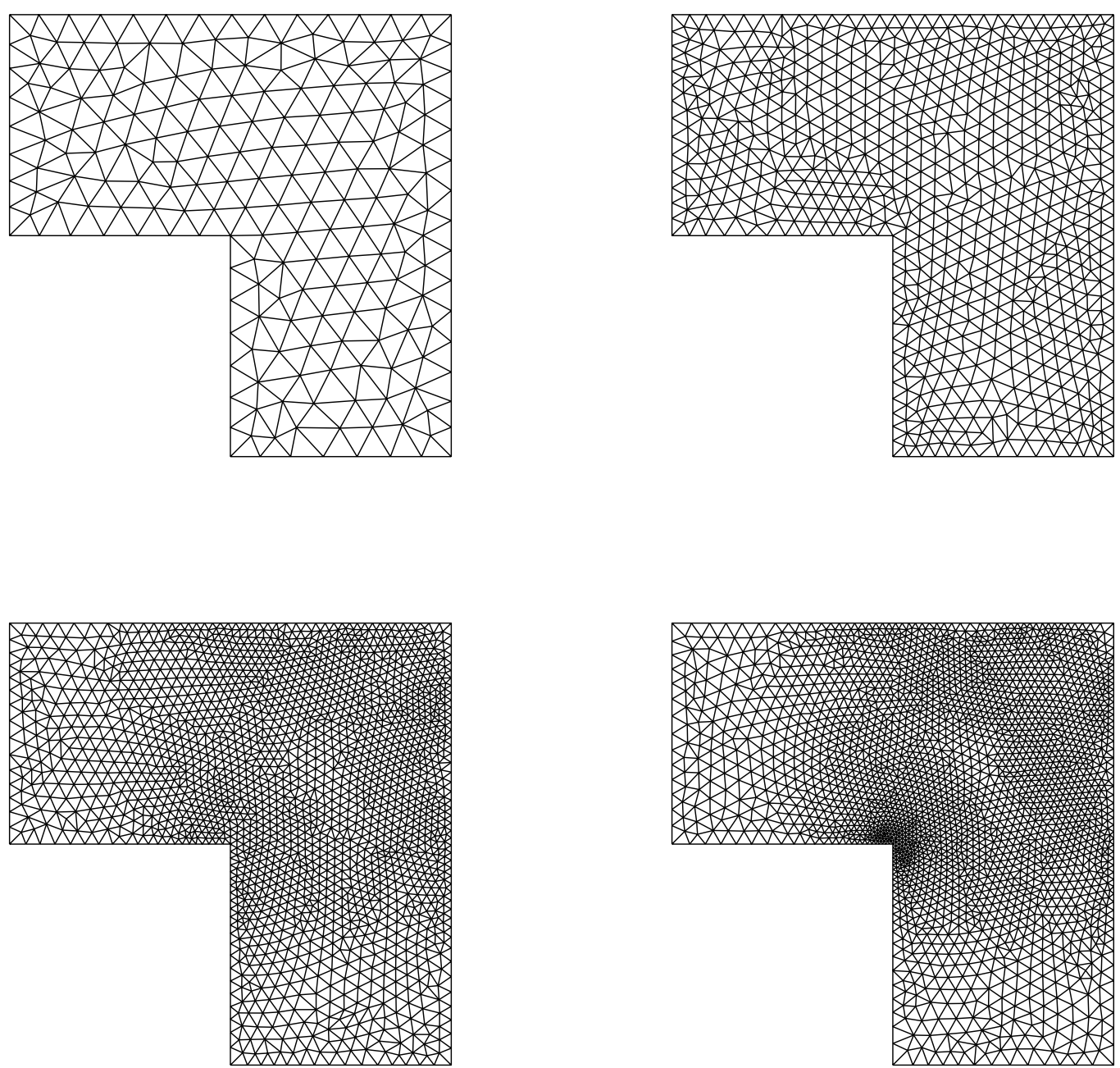

Figure 2: The sequence of adaptive mesh refinement for the smallest eigenvalue of the Stokes operator in the L-shaped domain. 


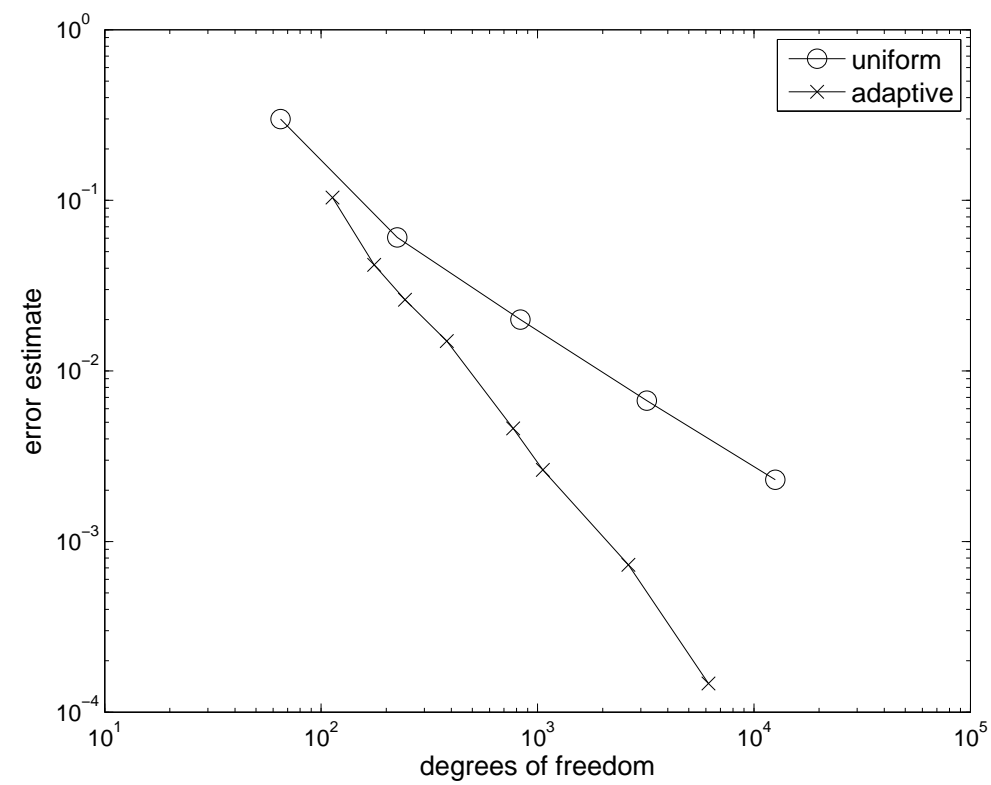

Figure 3: Error estimate for adaptive and uniform mesh refinement. 

(continued from the back cover)

A510 Janos Karatson, Sergey Korotov

Discrete maximum principles for FEM solutions of some nonlinear elliptic interface problems

December 2006

A509 Jukka Tuomela, Teijo Arponen, Villesamuli Normi On the simulation of multibody systems with holonomic constraints

September 2006

A508 Teijo Arponen, Samuli Piipponen, Jukka Tuomela

Analysing singularities of a benchmark problem

September 2006

A507 Pekka Alestalo, Dmitry A. Trotsenko

Bilipschitz extendability in the plane

August 2006

A506 Sergey Korotov

Error control in terms of linear functionals based on gradient averaging techniques

July 2006

A505 Jan Brandts, Sergey Korotov, Michal Krizek

On the equivalence of regularity criteria for triangular and tetrahedral finite element partitions

July 2006

A504 Janos Karatson, Sergey Korotov, Michal Krizek

On discrete maximum principles for nonlinear elliptic problems

July 2006

A503 Jan Brandts, Sergey Korotov, Michal Krizek, Jakub Solc On acute and nonobtuse simplicial partitions

July 2006

A502 Vladimir M. Miklyukov, Antti Rasila, Matti Vuorinen

Three sphres theorem for $p$-harmonic functions

June 2006 
HELSINKI UNIVERSITY OF TECHNOLOGY INSTITUTE OF MATHEMATICS RESEARCH REPORTS

The list of reports is continued inside. Electronical versions of the reports are available at $h$ ttp://math.tkk.fi/reports/ .

A516 Sergey Repin, Rolf Stenberg

Two-sided a posteriori estimates for the generalized stokes problem

December 2006

A515 Sergey Korotov

Global a posteriori error estimates for convection-reaction-diffusion problems

December 2006

A514 Yulia Mishura, Esko Valkeila

An extension of the L'evy characterization to fractional Brownian motion

December 2006

A513 Wolfgang Desch, Stig-Olof Londen

On a Stochastic Parabolic Integral Equation

October 2006

A512 Joachim Schöberl, Rolf Stenberg

Multigrid methods for a stabilized Reissner-Mindlin plate formulation

October 2006 